\title{
Orientaciones para una innovación en las bibliotecas públicas de la provincia de Segovia basada en las necesidades de información de los inmigrantes
}

\author{
Por María-Jesús Romera-Iruela
}

\begin{abstract}
Resumen: Las bibliotecas públicas son puertas de acceso a una sociedad del conocimiento igual para todos y escenarios idóneos para la integración social de los distintos colectivos de inmigrantes. Para ello tienen que crear servicios y promover proyectos que respondan a las demandas de las comunidades multiculturales a las que sirven. En este artículo se identifican las necesidades de información en las bibliotecas de los inmigrantes de la provincia de Segovia, a través de la elaboración de una serie de cuestionarios que han sido aplicados a informantes clave de tres fuentes diferentes pero complementarias: las bibliotecas públicas, los centros educativos y las asociaciones de inmigrantes. Se ofrecen orientaciones para una optimización innovadora en respuesta a las necesidades detectadas.

Palabras clave: Necesidades de información, Inmigrantes, Integración sociocultural, Innovación, Bibliotecas públicas.

Title: Overview of innovations at public libraries in Segovia province to meet the information needs of immigrants

Abstract: Public libraries are the entrance to a knowledge society with equal access for everyone and are also a suitable place for the social integration of many different groups of immigrants. Therefore, they should create services and initiate programs in response to the requests of the multicultural communities they serve. Segovia's libraries identified the information needs of immigrants through a series of questionnaires, administered through direct personal interactions with key informants from three distinct but complementary sources: public libraries, educational centres, and immigrant associations. We offer suggestions on innovative and optimal responses to the needs we detected.

Keywords: Information needs, Immigrants, Sociocultural integration, Innovation, Public libraries.

Romera-Iruela, María-Jesús. "Orientaciones para una innovación en las bibliotecas públicas de la provincia de Segovia basada en las necesidades de información de los inmigrantes". En: El profesional de la información, 2008, marzo-abril, v. 17, n. 2, pp. 155-164.

DOI: 10.3145/epi.2008.mar.04

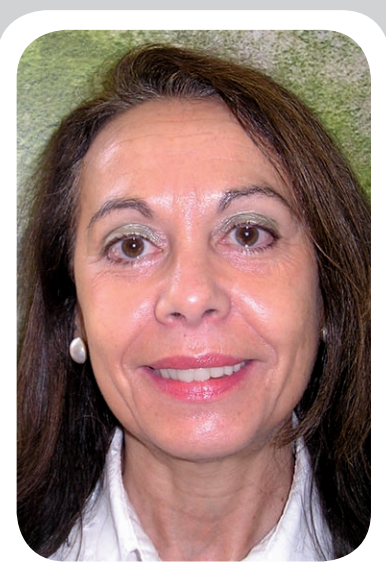

María-Jesús Romera-Iruela es doctora en ciencias de la educación por la Universidad Complutense de Madrid. Obtuvo los Premios extraordinarios de licenciatura y de doctorado. En los Premios nacionales de investigación e innovación educativa, obtuvo el tercer premio de tesis doctorales. Es profesora titular del Departamento de Teoría e Historia de la Educación de la Facultad de Educación en la Universidad Complutense y autora de diversos trabajos científicos. Sus principales líneas de investigación son: epistemología pedagógica, metodología de la investigación educativa y documentación pedagógica.
\end{abstract}

\section{Introducción}

La presencia creciente e intensa del fenómeno migratorio en la sociedad española ha conllevado que la multiculturalidad sea ya un rasgo propio. Este hecho trae consigo el reto de la integración de los colectivos lingüísticos y culturales que viven en ella. En la sociedad castellano leonesa dicho fenómeno adquiere gran significación puesto que a pesar de ser menor su cuantía, sus repercusiones demográficas, económicas y sociales son patentes, hasta el punto de que se ha afirmado que "el colectivo inmigrante supone una garantía de futuro para Castilla y León" (Roberto Martín, 2007). De ahí que se haya venido planteando la necesidad de estudiar su problemática así como también la de aplicar medidas ade- cuadas y soluciones eficaces y justas (Eugenio García, 2003). Los informes del Consejo Económico y Social de Castilla y León (2002, 2006), la Estrategia integral para la inmigración en Castilla y León 2004/2009 y el Plan integral de inmigración (2005-2009) de la Junta de Castilla y León son realizaciones en este sentido.

Las bibliotecas públicas son puertas de acceso a una sociedad de la información igual para todos y pueden ser agentes y escenarios idóneos para la integración socio-cultural de los inmigrantes mediante la creación de iniciativas y servicios que respondan a las necesidades de una comunidad diversa y también a través de programas desarrollados en colaboración con otras instancias sociales. 
Los proyectos bibliotecarios de integración han de estar basados en el conocimiento de las necesidades informativas y socioculturales de los inmigrantes. Como manifiesta Antonio Almagro (2006), es preciso abordar el problema en los contextos sociales, observando la inmigración "en vivo" -necesidades, demandas y aspiraciones de los inmigrantes-, teniendo en cuenta lo humano a través de sus plurales manifestaciones socioculturales.

\section{"Los proyectos bibliotecarios de integración han de estar basados en el conocimiento de las necesidades informativas y socioculturales de los inmigrantes"}

El propósito de este trabajo es dar a conocer uno de los objetivos de nuestro proyecto de investigación ${ }^{1}$ consistente en detectar las necesidades de información de los distintos colectivos de inmigrantes de la provincia de Segovia. Al ser limítrofe con la Comunidad de Madrid, nos permite conocer la incidencia de esta cercanía en los flujos migratorios. Dicha identificación se ha realizado desde la consideración de las tres perspectivas siguientes:

- Bibliotecas públicas, que nos van a dar a conocer las necesidades que detectan.

- Centros educativos, que nos refieren las necesidades de las comunidades culturales presentes en ellos.

- Asociaciones de inmigrantes, creadas por ellos mismos, que nos indican las de sus propios colectivos.

Nuestra pretensión es contribuir a los proyectos bibliotecarios de integración proporcionando el conocimiento de las necesidades de información de los inmigrantes y ofreciendo orientaciones sobre los servicios y programas que se precisan para que puedan ser satisfechas, favoreciendo así la toma de decisiones en materia de política bibliotecaria intercultural. En la línea del Plan integral de inmigración de la Junta de Castilla y León (2005), nuestro trabajo supone aportación para el logro de sus objetivos, entre ellos el de "favorecer el acceso a la cultura y a los recursos de información que poseen las bibliotecas (...) a la población inmigrante, creando las condiciones favorables para su integración" (p. 138).

\section{Antecedentes y fundamentación teórica}

En la Declaración de Copenhague se afirmó que las bibliotecas públicas, para responder a las cambiantes necesidades sociales, tienen que reconsiderar sus funciones y rediseñar sus servicios. Desde el año 1982, la Federación Internacional de Asociaciones e Instituciones Bibliotecarias (IFLA) viene trabajando en la investigación y el desarrollo de servicios bibliotecarios para poblaciones multiculturales. Fruto de estas actividades son sus normas y directrices elaboradas al objeto de que "todas las minorías étnicas, linguísticas y culturales dispongan de materiales bibliotecarios y de acceso a servicios de información conectados en red, en su idioma preferido, y que reflejen su propia cultura" (IFLA, p. 2). Se pretende así promover la comprensión y la tolerancia mutua entre todos los grupos culturales de la comunidad a la que sirve la biblioteca. En 1997 se crea su Comité de acceso a la información y libertad de expresión que ha elaborado la declaración sobre las bibliotecas y la libertad intelectual.

\section{"Existe la necesidad del acceso a los recursos de la mayor riqueza informativa, de manera que los miembros de una sociedad multicultural puedan progresar hacia una comprensión mutua más profunda"}

Este comité considera que "el acceso a la información no sólo contribuirá al entendimiento universal, sino que permite que la diversidad de opiniones sea reconocida y respetada, y mejora el mutuo enriquecimiento entre las diversas culturas" (Tony Evans, 1997, p. 2). Además la IFLA ha propuesto acciones para cumplir los principios afirmados del acceso a la información y la libertad de expresión y los ha colocado como fundamento de la práctica profesional, ofreciendo una perspectiva para configurar bibliotecas multiculturales. El Comité ejecutivo de esa federación ha aprobado y adoptado el texto del manifiesto de la biblioteca multicultural. Se espera que en octubre de 2008 se convierta en un manifiesto Unesco/IFLA (Kirsten Nielsen, 2007, p. 1).

En los planteamientos teóricos sobre el tema se constata un cambio. Con frecuencia, la necesidad de servicios bibliotecarios para las comunidades multiculturales ha sido descrita como la necesidad de que los colectivos minoritarios sean capaces de obtener materiales en sus propias lenguas y tradiciones culturales.

Una consideración más meditada enfatiza la necesidad del acceso a los recursos de mayor riqueza informativa, de manera que los miembros de una sociedad multicultural puedan progresar hacia una comprensión mutua más profunda. Este último enfoque está basado 
en la citada iniciativa de la IFLA sobre la libertad de expresión y de acceso a la información, que implica crear condiciones para que el acceso a la información no esté obstaculizado por prejuicios, concepciones falsas o competencias inadecuadas. Por ello esta iniciativa es potencialmente una contribución fundamental para el incremento de relaciones justas y armónicas en las comunidades multiculturales (Paul Sturges, 2005, p. 296). En el mismo sentido Ragnar Audunson (2005) ha afirmado que desarrollar la bibliotecología multicultural significa no sólo establecer servicios que se ajusten a las minorías étnicas y culturales sino también promover círculos en donde se encuentren las personas pertenecientes a culturas diferentes.

En cuanto a las realizaciones, las bibliotecas europeas, americanas y australianas han emprendido proyectos para la integración de los colectivos de inmigrantes presentes en sus comunidades. Algunos de los proyectos significativos son: Bazar (Noruega), Finfo (Dinamarca), Biblioteca multicultural (Finlandia), Queens (Nueva York), Biblioteca Pública de Toronto (Canadá), Open Road (Australia), Bibliotecas Interculturales (Andalucía) y Biblioteca Abierta (Madrid). El Grupo de Bibliotecas Catalanas asociadas a la Unesco también ha puesto en marcha experiencias de integración.

\section{"Las bibliotecas europeas, americanas y australianas han emprendido proyectos para la integración de los colectivos de inmigrantes presentes en sus comunidades"}

Los proyectos previos han implementado varias iniciativas según sus poblaciones, consistentes en colecciones multiculturales y servicios como cursos sobre las distintas lenguas, programas culturales, talleres y creación de portales electrónicos con información relativa a temas diversos y sobre la cultura del país.

\section{Metodología}

La configuración de la muestra de estudio (ver tabla 1) se realizó a partir de la consulta del Padrón Municipal de Habitantes, que permitió conocer la distribución de la población extranjera e inmigrante en la provincia de Segovia. Luego procedimos a la selección de aproximadamente un tercio de las bibliotecas públicas de dicha provincia. Los criterios utilizados han sido:

- Que fueran bibliotecas públicas del Estado, comunidad autónoma y municipales con convenio de su comunidad o vinculación al Centro Provincial Coordinador de Bibliotecas Públicas.

\begin{tabular}{|l|r|}
\hline Bibliotecas públicas & 5 \\
\hline Centros educativos & 10 \\
\hline Asociaciones de inmigrantes & 3 \\
\hline Informantes clave & 18 \\
\hline
\end{tabular}

Tabla 1. Muestra de estudio

- Que estuvieran ubicadas en los lugares que tienen la mayor cuantía y diversidad de inmigrantes.

Asimismo, se tuvo presente la mayor proximidad de los municipios con la Comunidad de Madrid.

El compromiso de anonimato adquirido en la investigación nos lleva a no desvelar los nombres de las cinco bibliotecas seleccionadas. Sin embargo, sí vamos a designar los lugares en donde ellas se encuentran: Segovia capital, Cantalejo, Cuellar, El Espinar y San Ildefonso.

Una vez fijadas dichas bibliotecas, seleccionamos el centro de educación infantil y primaria y el instituto de enseñanza secundaria que en dichos lugares tenían los mayores números de alumnos inmigrantes. En el caso de que sólo hubiera en el municipio un centro de alguno de los dos tipos, éste era incluido en la muestra. A través del Servicio de Atención a la Diversidad de Segovia pudimos obtener los datos que nos permitieron determinar los diez centros educativos que participaron en nuestra investigación.

Para la identificación de las 3 asociaciones de inmigrantes -creadas por ellos mismos-, realizamos una consulta a los ayuntamientos, a las ONGs de ayuda al inmigrante y a los departamentos de extranjería de los sindicatos.

\section{"Los 18 informantes clave son directores de bibliotecas públicas y de centros educativos, y presidentes de asociaciones de inmigrantes"}

Los informantes clave para cada una de las perspectivas objeto de estudio han sido: los directores de las bibliotecas públicas, los de los centros educativos y los presidentes de las asociaciones de inmigrantes. En total dieciocho. 
La recogida de datos se ha efectuado mediante cuestionarios. En su diseño tuvimos en cuenta las directrices de la IFLA, el Manifiesto de la Unesco sobre las bibliotecas públicas y los trabajos previos sobre estudios de usuarios y necesidades de información (Berger, 2002; Marcella; Baxter, 1999, 2000 y Petr, 2004, entre otros). Los cuestionarios, a pesar de una cierta diferenciación por sus destinatarios, tienen en común el campo para identificar las necesidades de materiales y servicios bibliotecarios. Antes de su aplicación se hizo una prueba piloto para determinar su comprensión y adecuación. Las preguntas en su mayor parte son estructuradas con opción libre. La cumplimentación de los formularios se realizó mediante interacción personal al objeto de poder maximizar la respuesta. Para este artículo se ha incluido como anexo a título de muestra, el campo "Necesidades de información" del cuestionario dirigido a las asociaciones.

El procedimiento seguido fue en síntesis el siguiente: establecimos contacto telefónico con todos los informantes clave con el propósito de darles a conocer la investigación y solicitar su colaboración en la misma. Aunque no suele ser lo usual en este tipo de estudios, gracias a nuestra previsión metodológica la respuesta de todos ellos fue de aceptación, por lo que a partir de junio de 2005 y de forma progresiva, establecimos una cita con ellos para proporcionarles la información que demandaran, darles a conocer el cuestionario y proceder a la aplicación. Todos ellos lo cumplimentaron, por lo que les agradecemos su colaboración.

\section{Principales resultados}

Ante la imposibilidad por límites de espacio de recoger todos los resultados obtenidos, presentamos a continuación algunos de los más significativos.

Existe un amplio acuerdo entre las bibliotecas públicas, los centros educativos y las asociaciones de inmigrantes en que estos colectivos tienen necesidad de disponer de un portal en internet con información sobre diferentes temas que sean de ayuda para su vida diaria y para su integración. Los porcentajes de acuerdo de dichas fuentes oscilan entre el $70 \%$ de los centros educativos con referencia a dicha necesidad para los padres de los escolares de las distintas culturas, y el 100\% según las asociaciones (gráfico 1).

Los seis temas sobre los que tienen mayor necesidad de información los inmigrantes son: 1) servicios sociales, 2) trabajo, 3) cursos de español para extranjeros, 4) permisos de residencia y de trabajo así como requisitos para obtener la nacionalidad y la reunificación familiar, 5) educación y 6) sanidad. Igualmente son citados por todas las fuentes aunque en menor medida, tanto cultura y ocio como derechos y obligaciones de los ciudadanos. La información sobre vivienda

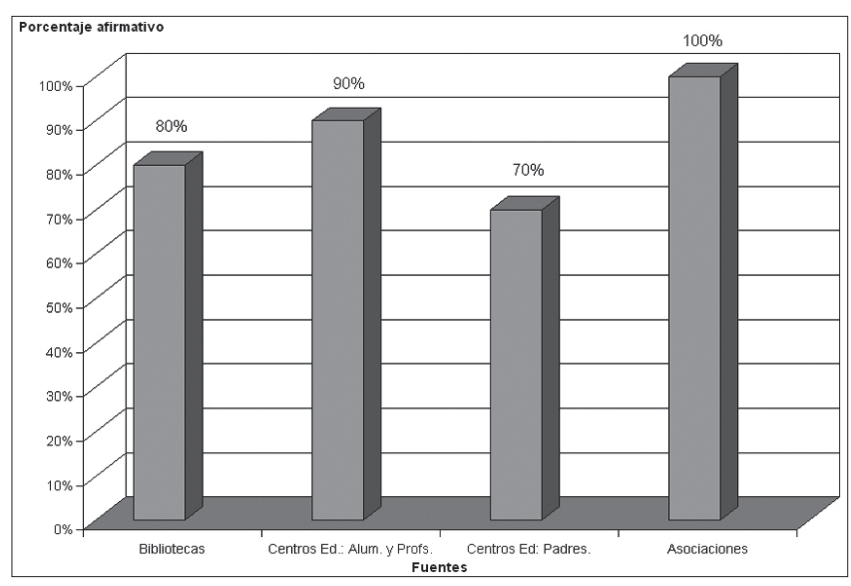

Gráfico 1: Necesidad de que las bibliotecas públicas tengan un portal informativo que facilite la integración de los inmigrantes

es fundamental para la totalidad de las asociaciones de inmigrantes (gráfico 2). Estos datos ponen de relieve la primacía de la información sobre las necesidades básicas por encima de la cultura, con la excepción del aprendizaje de la lengua del país de acogida.

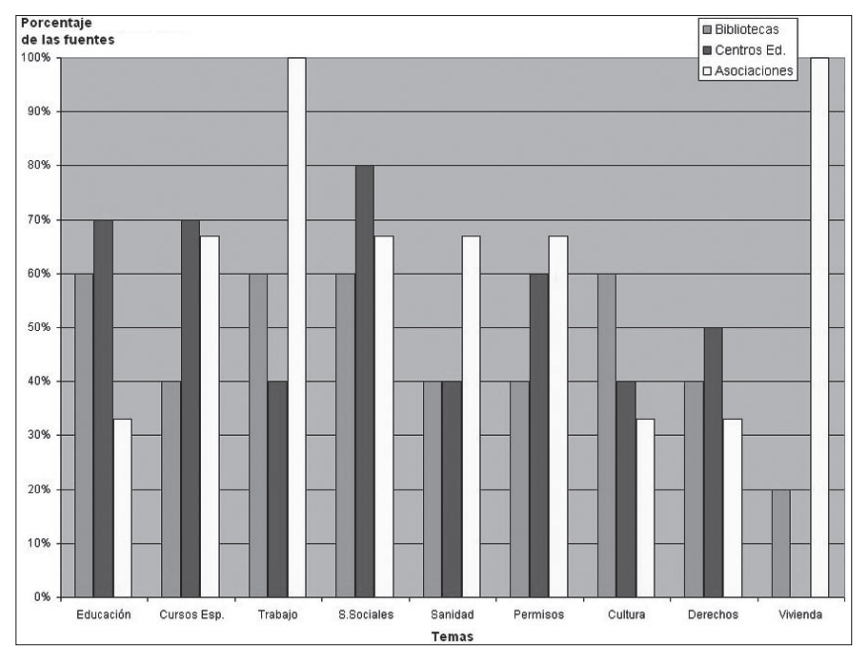

Gráfico 2: Temas sobre los que tienen mayor necesidad de información los inmigrantes

Las tres fuentes de información han señalado con una unanimidad casi total que los inmigrantes demandan una base de datos referencial sobre sus propias culturas que pueda ser consultada en sus lenguas maternas (gráfico 3).

En la determinación de los idiomas prioritarios, junto con los propios de España, en los que deberían estar los materiales en las bibliotecas públicas, la pauta de respuesta ha sido bastante convergente. El árabe y el búlgaro han sido los prioritarios, situándose en pie de igualdad, ya que así lo han considerado todas las fuentes de los tres tipos excepto una de cada uno de ellos. Les siguen en orden de importancia el inglés y el rumano. A continuación y desde la perspectiva de las bibliotecas y los centros educativos, se sitúan el francés y el polaco. 


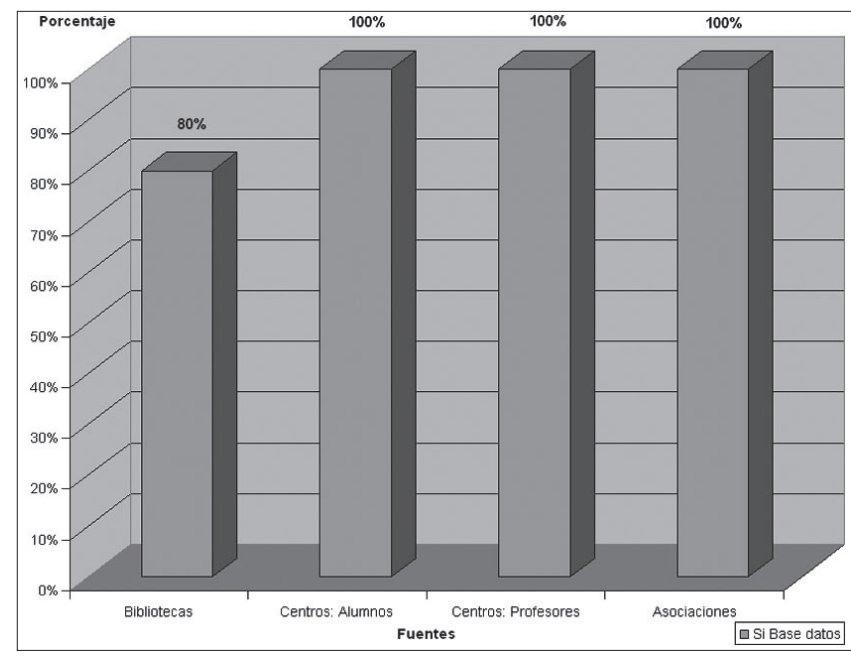

Gráfico 3: Necesidad de una base de datos referencial sobre las culturas de los inmigrantes y en sus propias lenguas pología de documentos, con pequeñas variaciones entre ellos.

La pauta de respuesta de los centros educativos y de las asociaciones de inmigrantes sobre los materiales que deberían tener las bibliotecas públicas para satisfacer ahora las necesidades de los escolares de las diferentes culturas en la enseñanza obligatoria, es bastante similar a la previa de los inmigrantes (ver gráfico 4). Constatamos igualmente el requisito de una amplia tipología documental para estos alumnos.

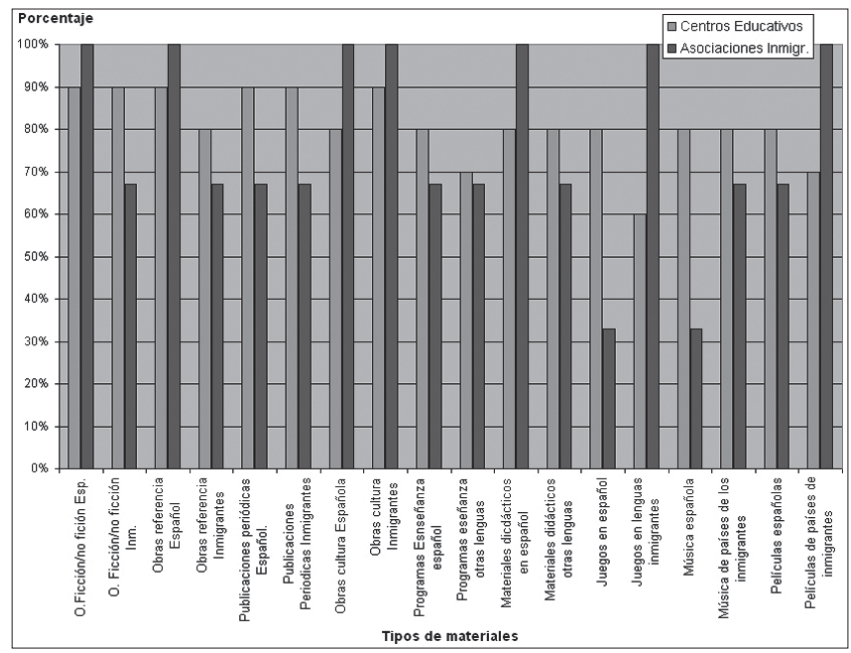

Gráfico 4: Materiales que deben tener las bibliotecas públicas para satisfacer las necesidades de los escolares de las diferentes culturas

La situación de las bibliotecas públicas desde el punto de vista de sus fondos no es acorde hasta el momento con las necesidades identificadas. Es preciso que la política bibliotecaria incida en la creación de colecciones sobre las culturas de los inmigrantes. Esta conclusión encuentra apoyo adicional en otro dato obtenido según el cual, el $60 \%$ de las bibliotecas que estiman que son pocos los inmigrantes que acuden a ellas, creen que se debe a los idiomas de sus fondos, la falta de conocimiento de su existencia y su falta de adecuación a las necesidades. Una asociación -que piensa que entre el 5 y el $24 \%$ de sus miembros hace uso de los servicios de la biblioteca, siendo esta cifra la menor de las indicadas-, coincide en parte al explicar el motivo de falta de conocimiento de las bibliotecas: las largas jornadas de trabajo y las ocupaciones familiares. Las asociaciones piensan que los inmigrantes irían con mayor frecuencia a las bibliotecas si en ellas hubiera más fondos de sus países.

\section{"Es preciso que la política bibliotecaria incida en la creación de colecciones sobre las culturas de los inmigrantes"}


Las tres fuentes consultadas consideran que los talleres culturales de las bibliotecas públicas son importantes para los alumnos de las diferentes culturas. Los más necesarios son los de cuenta cuentos, teatro, animación a la lectura y convivencia y conocimientos multiculturales. Destacamos que de éstos, el de animación a la lectura es señalado por la totalidad de las bibliotecas y de los centros educativos. A continuación se indican los talleres de poesía, escritura y títeres. Si comparamos la percepción de este requerimiento con la situación de las bibliotecas con respecto a dicho servicio, comprobamos que es preciso potenciar e incrementar la oferta de los talleres culturales citados.

Desde la perspectiva de las asociaciones y con unanimidad, los servicios bibliotecarios tradicionales más importantes para los inmigrantes son el préstamo de libros y la lectura en sala; a continuación se sitúa la consulta de periódicos y revistas. Dichos servicios se ofrecen en todas las bibliotecas.

Las asociaciones, las bibliotecas y los centros educativos, salvo en un caso referido a los padres de los alumnos, entienden que hacen falta otros servicios no tradicionales de las bibliotecas públicas para poder satisfacer a los inmigrantes en materia de información, educación y de ocio: acceso a portales electrónicos con información que ayude a su integración, internet, realización de actividades multiculturales e información laboral.

Además hay acuerdo total por parte de las asociaciones en la demanda de los talleres de ayuda en la realización de deberes escolares; son señalados por un $60 \%$ de las otras dos fuentes. Todas las bibliotecas consideran que importantes los servicios de formación para la utilización de los recursos de la biblioteca y para la utilización de las nuevas tecnologías de la información, este último también lo es en una amplia medida para las asociaciones. Por último los centros educativos estiman muy deseables los siguientes servicios específicos: visitas escolares a las bibliotecas públicas, apoyo y asesoramiento a los docentes en su tarea de fomento de la lectura, préstamo colectivo y orientación en la organización de la biblioteca escolar. Las primeras también tienen relevancia para las bibliotecas.

En el nivel de la prestación de servicios, el acceso a internet se ofrece en la mayoría de las bibliotecas consultadas siendo éste el que más utilizan los inmigrantes. También usan mucho, en una biblioteca que ha explicitado que lo ofrece, el taller de lectura y de animación a ella. Las asociaciones nos han indicado que algunos de sus miembros no poseen conocimientos de internet a nivel de usuario. Dos de ellas piensan que entre el 25 y el 50\% de estos acudirían a aprenderlos a las bibliotecas si se los ofertaran de forma gratuita. La otra asociación piensa que este porcentaje sería superior, entre el 51 y el 75\%. De ahí la necesidad de promover la alfabetización informativa en las bibliotecas.

Por último, la relación entre las bibliotecas públicas y escolares sigue siendo escasa. No obstante, los centros educativos, las asociaciones y las bibliotecas, con la excepción de una, entienden que la cooperación entre ellas es necesaria en forma de visitas escolares a la biblioteca pública, promoción de la lectura y la alfabetización, y programación en cooperación. Por otra parte la relación entre las bibliotecas públicas y las asociaciones de inmigrantes es prácticamente inexistente.

\section{"La relación entre las bibliotecas públicas y las asociaciones de inmigrantes es prácticamente inexistente"}

\section{Conclusiones. Algunas orientaciones para las bibliotecas}

De este estudio de las necesidades de información de los inmigrantes se deriva una serie de orientaciones para una optimización innovadora de las bibliotecas públicas de la provincia de Segovia. De forma sintética señalamos algunas de ellas:

- Las bibliotecas pueden favorecer la integración de los colectivos de inmigrantes presentes en la provincia mediante la creación de un portal electrónico que proporcione la mejor información sobre temas relativos a sus requerimientos más básicos, sobre cursos de español para extranjeros, sobre cultura y ocio así como sobre ciudadanía.

- La elaboración de una base de datos sobre las diferentes culturas es esencial para los inmigrantes y hace posible la comprensión mutua y la convivencia entre los miembros de esa comunidad a la que sirven las mencionadas bibliotecas.

- La política bibliotecaria tiene que favorecer la creación y el desarrollo de colecciones documentales mediante la incorporación de fondos de las culturas de los inmigrantes en una amplia tipología documental, de modo que las bibliotecas puedan prestar servicio a todas las personas del lugar, con independencia de su procedencia cultural. Además de las lenguas propias de España, el árabe y el búlgaro y luego el inglés y el rumano, son idiomas prioritarios para los fondos.

- El acercamiento de la población inmigrante a la biblioteca requiere acciones previas con el propósito de darla a conocer, así como una mayor adecuación a sus necesidades. 


\section{"El acercamiento de la población inmigrante a las bibliotecas requiere acciones previas con el propósito de darlas a conocer"}

- La potenciación de los talleres infantiles y servicios bibliotecarios que promuevan la convivencia y los conocimientos multiculturales es básica para fomentar la integración. Ambos pueden impulsarse mediante la cooperación entre las bibliotecas públicas y las escolares, con la participación de las asociaciones de inmigrantes.

- La elaboración de proyectos colaborativos entre las bibliotecas públicas y escolares para la promoción y fomento de la lectura, en los que haya implicación de los padres de los escolares y de las asociaciones, es una vía idónea para la implementación de dichos talleres dirigidos a los alumnos de todas las culturas. Esta acción puede inscribirse en el Plan de lectura de Castilla y León.

- Es preciso fomentar los servicios bibliotecarios no tradicionales relacionados con la información digital, incidiendo en el acceso a la misma y en la formación de usuarios en ese tipo de tecnologías.

Estas orientaciones, junto con otras implícitas en el diagnóstico efectuado, pueden ayudar a promover servicios interculturales en las bibliotecas públicas segovianas y son punto de partida para la creación de recursos de información. Su vinculación con otros estudios sobre las demandas de información de los inmigrantes les conferirá un mayor alcance.

Además esta investigación puede ser de ayuda y constituir una referencia para proyectos similares que se puedan plantear otras bibliotecas públicas españolas.

\section{Anexo: Campo "Necesidades de información" perteneciente al cuestionario para las asociaciones de inmigrantes}

7. Desde su punto de vista, ¿̇cuáles son los temas (con referencia al país de residencia) sobre los que tienen mayor necesidad de información las personas inmigrantes pertenecientes a la asociación? (máximo 6 temas)

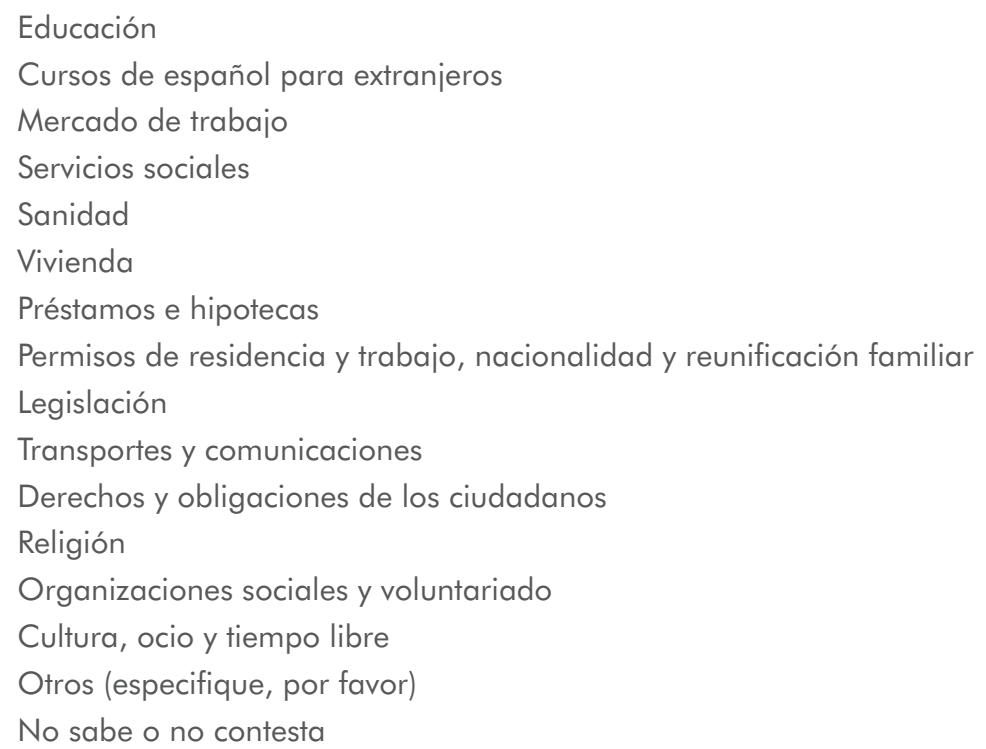

8. Desde su punto de vista, ¿̇sería útil, para las personas inmigrantes de su asociación, poder disponer de un sitio en internet que recoja información sobre temas en distintos ámbitos (educación, salud, servicios sociales, entre otros) que sean de interés para su vida diaria y para su integración social?

\section{Sí}

No

No sabe o no contesta

Si su respuesta ha sido No o No sabe/no contesta, pase directamente a la pregunta 10 
9. ¿Podría concretarnos algunos contenidos que considere que pueden ser objeto de consulta frecuente por muchas personas de la asociación si estuvieran accesibles a través de un portal en internet?

10. ¿Considera necesario, para las personas de su asociación, que las bibliotecas públicas tengan acceso a un portal de internet con información sobre temas de distintos ámbitos de la vida diaria que faciliten la integración social de los distintos colectivos de inmigrantes?

$$
\begin{aligned}
& \text { Sí } \\
& \text { No } \\
& \text { No sabe o no contesta }
\end{aligned}
$$

11. Desde su punto de vista, żlas personas que pertenecen a los diferentes colectivos de inmigrantes tienen necesidad de disponer de una base de datos con referencias sobre las diferentes culturas que pueda consultarse en sus propias lenguas?

\section{Sí}

No

$\square \quad$ No sabe o no contesta

12. Desde su punto de vista, ¿̇qué materiales deberían tener las bibliotecas públicas para poder satisfacer las necesidades (en materia de educación, información y ocio) de los diferentes colectivos de inmigrantes y las de los estudiantes de la enseñanza obligatoria, inmigrantes y españoles? (marque los que considere necesarios)

\section{Colectivos de inmigrantes}

Obras de ficción (literatura) y no ficción en lengua española (castellano)

Obras de ficción y no ficción en las lenguas maternas de los inmigrantes

Obras de referencia (enciclopedias, diccionarios, etc.) en español

Obras de referencia en las lenguas maternas de los inmigrantes

Revistas y periódicos en lengua española

Revistas y periódicos en las lenguas maternas de los inmigrantes

Materiales sobre la historia, la cultura y los desarrollos actuales de España

Materiales sobre la historia, la cultura y los desarrollos actuales de los países

de origen de los inmigrantes

Materiales didácticos en lengua española

Materiales didácticos en las lenguas maternas de los inmigrantes

Programas educativos para la enseñanza del español a inmigrantes

Programas educativos para la enseñanza de las lenguas de los inmigrantes

Juegos en lengua española

Juegos en las lenguas maternas de los inmigrantes

Discos y cintas de música española

Discos y cintas de música de los países de origen de los inmigrantes

Vídeo-películas españolas

Vídeo-películas de los países de origen de los inmigrantes

Otros materiales (especifique, por favor)
Estudiantes españoles e inmigrantes

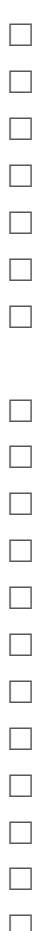

No sabe o no contesta 
13. Desde su punto de vista, además de las lenguas propias de España, ¿̇cuáles son los principales idiomas en los que deben estar los materiales en las bibliotecas públicas para poder satisfacer las necesidades de los distintos colectivos de inmigrantes que residen en la Comunidad Autónoma en la que se encuentra la asociación?

$\begin{array}{llll}\square & \text { Árabe } & \square & \text { Rumano } \\ \square \quad \text { Búlgaro } & \square & \text { Ruso } \\ \square \quad \text { Coreano } & \square & \text { Ucraniano } \\ \square \quad \text { Chino } & \square & \text { Otros idiomas (especifique, por favor) }\end{array}$

$\square \quad$ Francés

$\square \quad$ Inglés

$\square \quad$ Japonés

$\square \quad$ Polaco

$\square \quad$ Portugués

No sabe o no contesta

14. Indíquenos si considera necesario/s para los alumnos de la enseñanza obligatoria, inmigrantes y españoles, algún/ nos taller/res de actividades culturales de las bibliotecas públicas

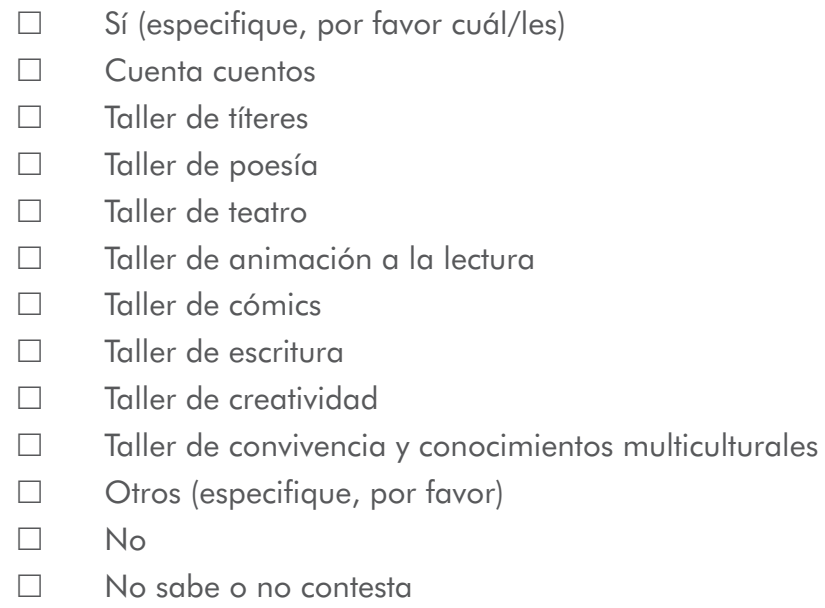

15. De los servicios tradicionales de las bibliotecas públicas, ¿̇díganos los cinco más importantes para las personas inmigrantes de su asociación con vistas a poder satisfacer sus necesidades en materia de educación, información y ocio?

Préstamo de libros

Préstamo de discos y cintas de música

Préstamo de video-películas

Consulta de periódicos y revistas

Servicio de información bibliográfica y de orientación documental

Lectura en sala

Otros servicios tradicionales (especifique, por favor)

No sabe o no contesta

16. Díganos si considera necesarios otros servicios no tradicionales de las bibliotecas públicas para satisfacer las necesidades de las personas inmigrantes de su asociación

Sí (especifique cuál/es, por favor)

Acceso a portales de Internet con información sobre temas que ayuden a su integración

Cursos de español para extranjeros

Cursos sobre las lenguas de las diferentes culturas

Talleres de ayuda en la realización de deberes escolares

Formación para la utilización de los recursos de la biblioteca

Formación para la utilización de las nuevas tecnologías de la información 
Apoyo a programas de alfabetización y a la elaboración de materiales de aprendizaje de las lenguas de las diferentes culturas

Acceso a Internet

Servicio de información laboral

Servicio de información sobre ocio y tiempo libre

Realización de actividades de ocio y tiempo libre

Realización de actividades multiculturales

Otros (especifique, por favor)

No

No sabe o no contesta

17. Desde su punto de vista, ¿̇qué medios utilizan las personas inmigrantes de su asociación para satisfacer sus necesidades de información (incluidas las referidas a educación y ocio)?

Asociaciones y ONGs
Ayuntamiento
Bibliotecas
Internet
Prensa
Radio
Televisión
Otros medios (especifique, por favor)
No sabe o no contesta

\section{Nota}

1. Proyecto financiado por el Ministerio de Ciencia y Tecnología en el marco del Plan Nacional de I+D+I 2000-2003. Referencia BFF2003-05157.

\section{Bibliografía}

Almagro, Antonio. "La educación intercultural en la diversidad multicultural. Implicaciones y estrategias de actuación". En: Anales de historia contemporánea, 2006, n. 22, pp. 167-176.

Audunson, Ragnar. "The public library as a meeting-place in a multicultural and digital context”. En: Journal of documentation, 2004, v. 61, n. 3, pp. $429-441$.

Berger, Agot. "Recent trends in library services for ethnic minorities-the Danish experience". En: Library management, 2002, v. 23, n. 1-2, pp. 79-87.

Consejo Económico y Social de Castilla y León. La población inmigrante en Castilla y León (documento técnico). Consultado en: 04-10-07.

http://www.cescyl.es/informes/iniciativapropia.php

Consejo Económico y Social de Castilla y León. La inmigración en Castilla y León tras los procesos de regularización: aspectos poblacionales y jurídicos (parte 1). La nueva inmigración en Castilla y León (parte 2). Consultado en: 04-10-07.

http://www.cescyl.es/informes/iniciativapropia.php

Evans, Tony. Informe preparado para la reunión del consejo de la IFLA en Copenhague, Dinamarca. Consultado en: 10-11-06.

http://www.infla.org/IV/infa63caifs.htm

García, Eugenio. "La inmigración en Castilla y León a comienzos del siglo XXI: análisis, problemática y perspectivas". En: Papeles de geografía, 2003, enero-junio, n. 37, pp. 77-104.

International Federation of Library Associations and Institutions. Comunidades multiculturales. Directrices para el servicio bibliotecario. Consultado en: 10-11-06. http://www.ifla.org

Junta de Castilla y León. Plan integral de inmigración en Castilla y León 2005-2009. Consultado en: 04-10-07.

http://centros.educa.jcyl.es/creivalladolid/upload/plan_integral.pdf

Marcella, Rita; Baxter, Graeme. "The information needs and the information seeking behaviour of a national sample of the population in the United Kingdom, with special reference to needs related with citizenship". En: Journal of documentation, 1999, v. 55, n. 2, pp. 159-183.

Marcella, Rita; Baxter, Graeme. "Citizenship information needs in the UK: results of a national survey of the general public by personal doorstep interview". En: Aslib proceedings, 2000, v. 52, n. 3, pp. 115-123.

Martín, Roberto. Entrevista con Félix Colsa, responsable de Inmigración de la Junta de Castilla y León. Consultado en: 19-11-07.

http://www.robertomartinwordpress.com

Nielsen, Kirsten Leth. "Chair's column”. En: IFLA newsletter. Library services to multicultural populations, 2007, May, n. 2, pp. 1-1.

Petr, Kornelija. "Information needs of the Romany minority in Eastern Croatia: pilot study". En: New library world, 2004, v. 105, n. 9-10, pp. 357369

Sturges, Paul. "Understanding cultures, and IFLA's freedom of access to information and freedom of expression (Faife) core activity". En: Journal of documentation, 2005, v. 61, n. 2, pp. 296-305.

María-Jesús Romera-Iruela, Departamento de Teoría e Historia de la Educación, Facultad de Educación, Universidad Complutense, Rector Royo Villanova $s / n$, 28040 Madrid.

mjromera@edu.ucm.es 